\title{
Adesão ao Tratamento Farmacológico de Indivíduos com Diabetes Cadastrados no Hiperdia em uma Unidade de Saúde Baiana
}

\author{
Eliziane Mallane Rosa de Sá, ${ }^{1}$ Pâmala Évelin Pires Cedro, ${ }^{2}$ \\ Tatilla Putumuju Santana Mendes, ${ }^{1}$ Alana Caise dos Anjos Miranda, ${ }^{3}$ \\ Gildomar Lima Valasques Junior, ${ }^{1}$ Danyo Maia Lima ${ }^{1}$
}

\begin{abstract}
RESUMO
O Diabetes Mellitus é um problema de saúde pública que provoca inúmeras internações. Este estudo avaliou a adesão ao tratamento medicamentoso de indivíduos com diabetes cadastrados no programa Hiperdia em uma unidade de saúde do município de Jequié-Ba. Trata-se de um estudo quantitativo, descritivo analítico e de corte transversal, que foi desenvolvido no Centro de Saúde Almerinda Lomanto e contou com 81 pacientes. Um formulário referente aos aspectos sociodemográficos, hábitos de vida e aspectos clínicos da doença foi aplicado, além dos testes de Morisky e Batalla, que avaliaram a adesão ao tratamento medicamentoso. A análise dos dados foi realizada pelo programa SPSS. A associação entre a adesão e as variáveis independentes foi analisada pelo teste de qui-quadrado $\left(X^{2}\right)$, a hipótese de associação foi aceita quando a probabilidade fosse $\leq 0,05$. O perfil sociodemográfico demonstrou a maior prevalência de mulheres, idosos, solteiros, aposentados, com Ensino Fundamental incompleto e renda de 1/2 salários mínimos. A adesão foi observada em $40,7 \%$ dos participantes, pelo teste de Batalla e $51,9 \%$ pelo teste de Morisky. Quanto à associação estatística, a adesão pelo teste de Morisky apresentou significância quando relacionada à renda, porém, pelo teste de Batalla, apenas a variável tabagismo foi significante. Não houve associação significativa entre as demais variáveis em ambos os testes. Os pacientes atendidos no Centro de Saúde relataram dificuldade em aderir à terapêutica medicamentosa para diabetes, principalmente por falta de informação sobre a doença e o tratamento, ressaltando a importância da comunicação dos pacientes com os profissionais de saúde.
\end{abstract}

Palavras-chave: Diabetes mellitus; saúde pública; Hiperdia; farmacoterapia.

\section{ADHERENCE TO PHARMACOLOGICAL TREATMENT OF INDIVIDUALS}

WITH DIABETES REGISTERED AT HIPERDIA IN A BAHIAN HEALTH UNIT

\section{ABSTRACT}

Diabetes Mellitus is a public health problem that causes numerous hospitalizations. This study evaluated the adherence to drug treatment of individuals with diabetes registered in the Hiperdia program at a health unit in the municipality of Jequié-Ba. This is a quantitative, descriptive analytical and cross-sectional study, which was developed at the Almerinda Lomanto Health Center and involved 81 patients. A form referring to sociodemographic aspects, lifestyle and clinical aspects of the disease was applied, in addition to the Morisky and Batalla tests, which assessed adherence to drug treatment. Data analysis was performed using the SPSS program. The association between adherence and independent variables was analyzed using the chi-square test $\left(\mathrm{X}^{2}\right)$, the association hypothesis was accepted when the probability was $\leq 0.05$. The sociodemographic profile showed the highest prevalence of women, elderly, single, retired, with incomplete elementary education and income of 1-2 minimum wages. Adherence was observed in $40.7 \%$ of participants, using the Batalla test and $51.9 \%$ using the Morisky test. As for the statistical association, adherence by the Morisky test was significant when related to income, however, by the Batalla test, only the smoking variable was significant. There was no significant association between the other variables in both tests. Patients seen at the Health Center reported difficulty in adhering to medication therapy for diabetes, mainly due to the lack of information about the disease and treatment, emphasizing the importance of patients' communication with health professionals.

Keywords: Diabetes mellitus; public health; Hiperdia; pharmacotherapy.

RECEBIDO EM: 23/9/2020

MODIFICAÇÕES SOLICITADAS EM: 26/5/2021

ACEITO EM: 24/6/2021

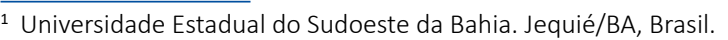

${ }^{2}$ Autora correspondente. Universidade Estadual do Sudoeste da Bahia. Avenida José Moreira Sobrinho - Jequiezinho. Jequié/BA, Brasil. CEP 45208-409. http://lattes.cnpq.br/8594368617413348. https://orcid.org/0000-0002-2888-1140. pamalaevelinpires@hotmail.com

${ }^{3}$ Universidade Estadual de Feira de Santana (UEFS). Feira de Santana/BA, Brasil.
} 


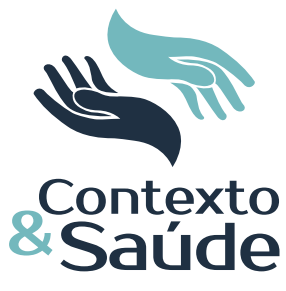

\section{INTRODUÇÃO}

O Diabetes Mellitus (DM) é um problema de saúde pública, prevalente em todos os países, independentemente do seu grau de desenvolvimento. ${ }^{1} \mathrm{O} \mathrm{DM} \mathrm{e}$ suas complicações são responsáveis por grande consumo de recursos de saúde, por necessidade de um elevado investimento por parte dos governos em consultas, exames complementares de diagnóstico, medicamentos, soluções tecnológicas e tratamentos de complicações agudas e crônicas. ${ }^{2}$

Estima-se que em cerca de 15 anos o DM ocupará o sétimo lugar no ranking das causas de mortes no Brasil, o que demonstra tratar-se de uma das doenças epidêmicas mais importantes do século. ${ }^{3}$ Em 2019 a Federação Internacional de Diabetes relatou cerca de 463 milhões de pessoas acometidas por diabetes. Se as tendências atuais persistirem, o número de pessoas com diabetes poderá ser superior a 700 milhões em 2045 em todo o mundo e cerca de 26 miIhões no Brasil. ${ }^{4}$

A partir da compreensão de que o DM compõe uma das primeiras causas de hospitalizações no sistema público, o Ministério da Saúde do Brasil tem adotado estratégias e ações na tentativa de reduzir o número de hospitalizações e de atingir o acompanhamento e o tratamento adequados na atenção básica. Entre essas ações destaque-se o Plano de Reorganização da Atenção à Hipertensão Arterial (HA) e ao Diabetes Mellitus, que prevê o cadastro e o acompanhamento dos usuários portadores de HA e/ou DM por meio do Sistema de Cadastramento e Acompanhamento de Hipertensos e Diabéticos (HIPERDIA). ${ }^{5}$

O DM é uma doença metabólica crônica caracterizada pelo aumento anormal da glicose no sangue, como consequência de prejuízos na produção ou ação de insulina secretada pelas células beta no pâncreas. ${ }^{6}$ Tal patologia está associada ao aumento da mortalidade e ao alto risco de desenvolvimento de complicações que tradicionalmente são categorizadas como distúrbios microvasculares e macrovasculares, que resultam, principalmente, em retinopatia, nefropatia, neuropatia, doença coronariana, doença cerebrovascular e doença arterial periférica. ${ }^{7}$

Entre os tipos de DM existentes, os mais comuns são o diabetes tipo 1 (DM1) e diabetes tipo 2 (DM2). A DM1 caracteriza-se pela destruição autoimune, parcial ou total, das células beta pancreáticas, resultando na ausência progressiva da produção de insulina. A DM2 é o tipo de diabetes mais comum entre os casos de diabéticos. ${ }^{8}$

O tratamento para DM tem como meta a normoglicemia, e por isso deve incluir um conjunto de medidas que visem a melhorar a atividade da insulina e diminuir os níveis de glicose circulantes. Para pacientes com DM1, consiste no tratamento insulínico, devendo ser instituído logo após o diagnóstico, e para pacientes com DM2 o tratamento não farmacológico é um dos pilares fundamentais na assistência global, mas pode ser acompanhada do tratamento farmacológico por um agente antidiabético oral, e também incluir o uso da insulina. ${ }^{9}$

A principal causa do surgimento das complicações nos indivíduos com diabetes é a dificuldade em aderir à terapêutica farmacológica, que inclui o custo de medicamentos, efeitos adversos, grau de informação e compreensão, dificulda-

Editora Unijuí - Revista Contexto \& Saúde - ISSN 2176-7114 - v. 21, n. 44, out./dez. 2021 
de de acesso aos serviços de saúde e o caráter assintomáticos da doença. ${ }^{10}$ Algumas condições de saúde exigem maior cuidado e a não adesão ao tratamento de doenças crônicas pode levar a um pior prognóstico da doença.

Considerando o cenário do DM como crescente problema de saúde pública e suas complicações que comprometem a saúde dos pacientes, surge a necessidade em compreender as variáveis relacionadas com a adesão da terapêutica de pacientes diabéticos, para reunir informações suficientes que possibilitem o desenvolvimento de medidas educativas e estratégias de intervenções mais eficientes para o controle do DM. Nesse sentido, este estudo buscou avaliar os aspectos envolvidos com a adesão ao tratamento medicamentoso de indivíduos com diabetes cadastrados no programa Hiperdia em uma unidade de saúde do município de Jequié-Ba.

\section{METODOLOGIA}

Trata-se de um estudo quantitativo, descritivo analítico e de corte transversal, que foi conduzido no municio de Jequié, no Estado da Bahia. O cenário de estudo foi o Centro de Saúde Almerinda Lomanto, que abrange 48 microáreas com 4.030 famílias cadastradas e 629 indivíduos com diabetes atendidos.

Foram empregados como critério de inclusão: pacientes portadores de DM1 ou DM2; cadastrados na unidade no programa Hiperdia de ambos os sexos; possuir idade $\geq 18$ anos, não havendo idade limite superior, que estivessem agendados para o atendimento com a enfermeira no mês da coleta. Foram adotados como critérios de exclusão: pacientes que não usavam medicamentos e aqueles que se negaram a assinar o Termo de Consentimento Livre e Esclarecido (TCLE).

A coleta de dados foi realizada por meio de aplicação de formulários estruturados, realizados uma única vez, durante a espera das avaliações, realizadas periodicamente por uma profissional de enfermagem e agendadas previamente pelos pacientes cadastrados na unidade, no mês de agosto de 2018. O formulário envolveu dados sociodemográficos, hábitos de vida, tratamento e aspectos clínicos da doença.

A aplicação dos formulários teve início após a leitura, compreensão e assinatura do TCLE pelo entrevistado.

A variável dependente adesão dos usuários foi avaliada pelos testes padronizados de Batalla ${ }^{11}$ e Morisky ${ }^{12}$ adaptado de maneira independente. $O$ teste Batalla foi composto por 3 (três) perguntas e o teste de Morisky por 4 (quatro) perguntas. Os testes mensuraram a adesão por meio do conhecimento dos usuários sobre sua doença e pelas atitudes dos pacientes quanto ao uso dos medicamentos.

Como variáveis independentes foram avaliados aspectos relacionados ao paciente, incluindo dados sociodemográficos (sexo, idade, estado civil, escolaridade, ocupação e renda familiar); hábitos de vida (tabagismo, consumo de bebida alcoólica e atividade física); tratamento e aspectos clínicos (como comparecimento aos retornos às consultas com a enfermeira, comorbidades, complicações 
do diabetes, nome e posologia dos medicamentos hipoglicemiantes em uso; forma de obtenção dos medicamentos).

O processamento e análise dos dados foram realizados utilizando o programa SPSS Statistics for Windows (IBM SPSS. 21.0, 2012, Armonk, NY: IBM Corp) e os gráficos gerados a partir do Software Graphpad Prism 5.0. A análise dos dados envolveu a aplicação de estatística descritiva, mediante a distribuição das frequências (absolutas e relativas) em todas as variáveis e foram apresentadas a média e o desvio padrão apenas da variável idade.

A estatística inferencial compreendeu a análise de associação das variáveis: sexo, idade, estado civil, escolaridade, ocupação, renda, residentes no domicílio, tabagismo, alcoolismo e atividade física (variáveis independentes) com a adesão terapêutica (variável dependente) segundo a ferramenta de Morisky e Batalla. Para tanto empregou-se o teste Qui-quadrado (x2) com o intervalo de confiança de $95 \%$ em todas as análises. Considerou-se aceita a hipótese de associação quando a probabilidade fosse $\leq 0,05(p<0,05)$.

O estudo foi aprovado pelo Comitê de Ética em Pesquisa da Universidade Estadual do Sudoeste da Bahia (CEP-UESB) sob CAAE: 92450918.0.0000.0055, número do Parecer: 2.783 .593 e atende ao disposto na Resolução 466/2012 do Conselho Nacional de Saúde.

\section{RESULTADOS}

Dos 629 pacientes cadastrados no programa Hiperdia da unidade de saúde, 128 foram agendados entre os dias 6 a 30 de agosto para a consulta com a enfermeira, mas apenas 99 compareceram, e destes 3 foram excluídos por não utilizarem medicamentos e 15 não aceitaram responder ao formulário, portanto a amostra final constituiu-se de 81 indivíduos.

Os pacientes apresentavam faixa etária entre 31 e 96 anos, média de idade de $60,9( \pm 12,2)$, e destes $75,3 \%$ do sexo feminino. Prevaleceram idosos com faixa etária $\geq 60$ anos, solteiros, aposentados, Ensino Fundamental incompleto e possuindo renda de 1 a 2 salários mínimos. Quanto aos hábitos de vida, $60,5 \%$ não praticavam exercício físico, o consumo de tabaco e bebida alcoólica foi mencionado em 3,7\% e 2,5\% dos entrevistados, respectivamente. A maior parcela dos pacientes relatou retornar à consulta com a enfermeira todo mês. As características sociodemográficas e de hábitos de vida estão apresentadas na Tabela 1.

A Figura 1 apresenta os resultados relacionados às comorbidades mencionadas entre os entrevistados. Além do diabetes, $80,2 \%$ dos pacientes apresentam outras comorbidades, sendo que destes $93,8 \%$ apresentam hipertensão arterial (HA), 29,2\% hipercolesterolemia (HC) e 23,1\% possuíam ambas as comorbidades. Com relação às complicações da doença $9,9 \%$ dos pacientes relataram presença. 
Tabela 1 - Distribuição dos usuários assistidos pelo Programa Hiperdia segundo as variáveis socioeconômicas

\begin{tabular}{|c|c|c|c|}
\hline Variáveis & & $\mathbf{N}$ & (\%) \\
\hline \multirow[t]{2}{*}{ Sexo } & Feminino & 61 & 75,3 \\
\hline & Masculino & 20 & 24,7 \\
\hline \multirow[t]{3}{*}{ Idade } & $30-49$ anos & 12 & 14,8 \\
\hline & $50-59$ anos & 24 & 29,6 \\
\hline & 60 anos ou mais & 45 & 55,5 \\
\hline \multirow[t]{4}{*}{ Estado civil } & Solteiro & 33 & 40,7 \\
\hline & Casado & 24 & 29,6 \\
\hline & Viúvo & 16 & 19,8 \\
\hline & Separado & 2 & 2,5 \\
\hline \multirow[t]{5}{*}{ Escolaridade } & Analfabeto & 16 & 19,8 \\
\hline & Lê e escreve & 7 & 8,6 \\
\hline & Ensino Fund. Incompleto & 45 & 55,6 \\
\hline & Ensino Fund. Completo & 6 & 7,4 \\
\hline & Outros & 7 & 8,6 \\
\hline \multirow[t]{4}{*}{ Ocupação } & Aposentado & 45 & 55,6 \\
\hline & Do lar & 20 & 24,7 \\
\hline & Doméstica & 4 & 4,9 \\
\hline & Outros & 12 & 14,8 \\
\hline \multirow[t]{3}{*}{ Renda } & < 1 salário mínimo & 9 & 11,1 \\
\hline & 1- 2 salários mínimos & 67 & 82,7 \\
\hline & $>3$ salários mínimos & 5 & 6,2 \\
\hline \multirow[t]{2}{*}{ Residentes no domicílio } & 1 a 3 & 62 & 76,5 \\
\hline & 4 a 6 & 17 & 21,0 \\
\hline \multirow[t]{3}{*}{ Cuidador dos medicamentos } & Paciente & 78 & 96,3 \\
\hline & Filhos & 2 & 2,5 \\
\hline & Outros & 1 & 1,2 \\
\hline \multirow[t]{2}{*}{ Fumantes } & $\operatorname{Sim}$ & 3 & 3,7 \\
\hline & Não & 78 & 96,3 \\
\hline \multirow[t]{2}{*}{ Consumo de bebida alcoólica } & Sim & 2 & 2,5 \\
\hline & Não & 79 & 97,5 \\
\hline \multirow[t]{2}{*}{ Atividade física } & Sim & 32 & 39,5 \\
\hline & Não & 49 & 60,5 \\
\hline \multirow[t]{2}{*}{ Retornos médicos } & Mensalmente & 80 & 98,8 \\
\hline & Semestralmente & 1 & 1,2 \\
\hline
\end{tabular}

Fonte: Elaborada pelos autores (2018) 
Figura 1 - Comorbidades apresentadas pelos pacientes

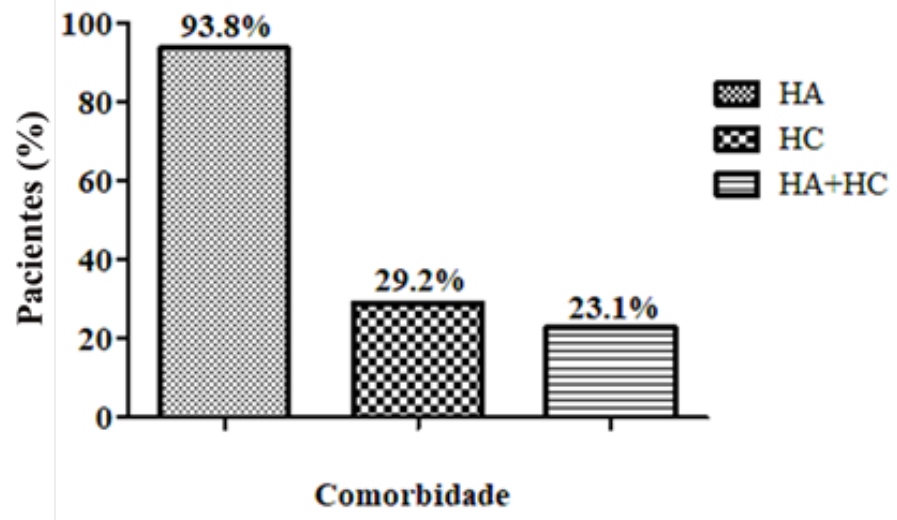

Fonte: Elaborada pelos autores, 2018.

Entre os entrevistados, $64,2 \%$ conhecem e sabem o nome do medicamento utilizado; quanto ao uso, $40,7 \%$ fazem o uso duas vezes ao dia do medicamento para diabetes, $80,2 \%$ fazem uso de outros medicamentos e $72,8 \%$ dos medicamentos utilizados são adquiridos nas farmácias. As demais características relacionadas ao tratamento e aspectos clínicos estão apresentadas na Tabela 2.

Tabela 2 - Distribuição dos usuários assistidos pelo Programa Hiperdia segundo as variáveis relacionadas ao tratamento e aspectos clínicos

\begin{tabular}{|l|c|c|c|}
\hline Variáveis & N & (\%) \\
\hline Comorbidades & Sim & 65 & 80,2 \\
\cline { 2 - 4 } & Não & 16 & 19,8 \\
\hline \multirow{2}{*}{ Complicações } & Sim & 8 & 9,9 \\
\hline Medicamentos utilizados & Não & 73 & 90,1 \\
\cline { 2 - 4 } & Sabe & 52 & 64,2 \\
\hline \multirow{3}{*}{ Uso de medicamento ao dia } & Não sabe & 29 & 35,8 \\
\cline { 2 - 4 } & Uma vez & 17 & 21,0 \\
\cline { 2 - 4 } & Duas vezes & 33 & 40,7 \\
\cline { 2 - 4 } & Três vezes & 30 & 37,0 \\
\hline \multirow{3}{*}{ Outros medicamentos } & Quatro vezes & 1 & 1,3 \\
\hline \multirow{2}{*}{ Onde adquire } & Sim & 65 & 80,2 \\
\cline { 2 - 4 } & Não & 31 & 19,8 \\
\cline { 2 - 4 } & Posto & 11 & 13,6 \\
\cline { 2 - 4 } & Farmácia & 59 & 72,8 \\
\cline { 2 - 4 } & Outros & 11 & 13,6 \\
\hline
\end{tabular}

Fonte: Elaborada pelos autores (2018)

Entre os indivíduos com diabetes, os medicamentos mais consumidos foram Cloridrato de metformina, Glibenclamida, com uma associação entre eles em 39,5\%, e insulina, caracterizando uma amostra de pacientes com DM2, como mostra na Figura 2. 
Figura 2 - Medicamentos hipoglicemiantes utilizados pelos pacientes entrevistados

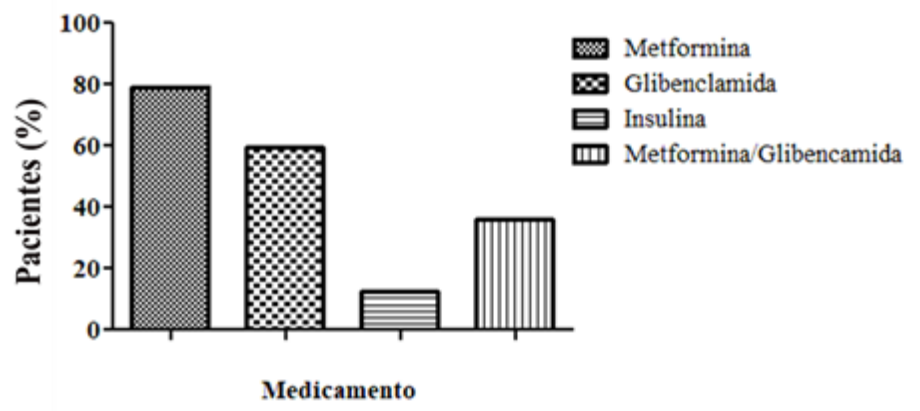

Fonte: Elaborada pelos autores (2018).

Os medicamentos mais citados quanto ao uso entre os 65 pacientes com diabetes, além dos hipoglicemiantes orais, foram: Losartana, Hidroclorotiazida, Sinvastatina, Atenolol, Anlodipino, Enalapril e Captopril, como demostrado na Figura 3.

Figura 3 - Outros medicamentos utilizados pelos pacientes
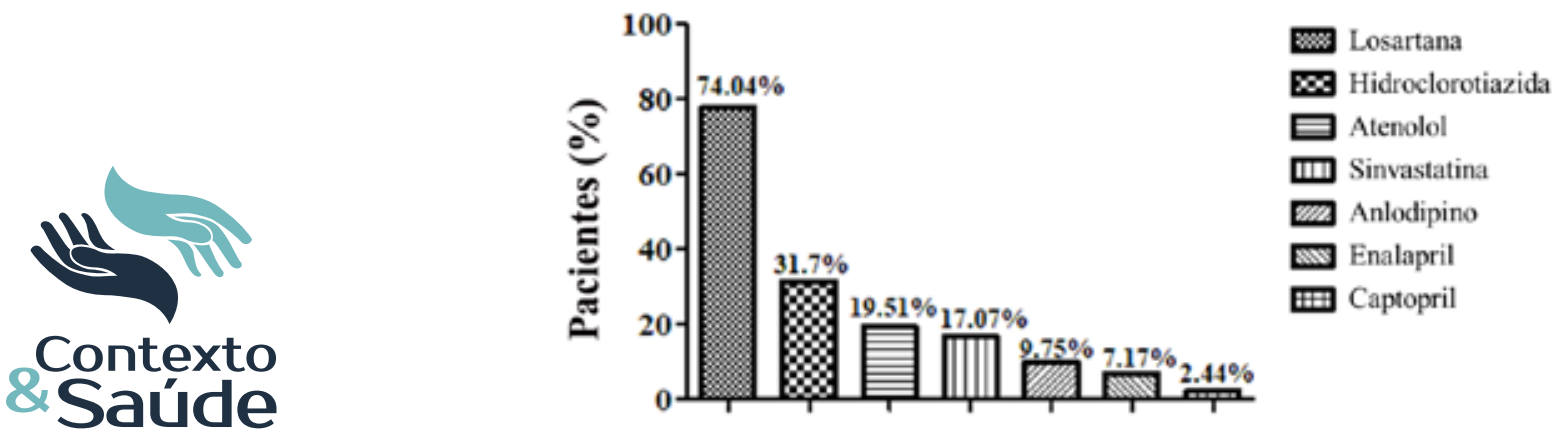

Medicamento

Fonte: Elaborada pelos autores (2018)

Os resultados dos testes de Batalla e Morisky são apresentados no Quadro 1 e Quadro 2, respectivamente. Segundo o conhecimento dos entrevistados sobre sua doença, a maioria respondeu corretamente as duas primeiras perguntas, $75,3 \%$ e 91,4\%, respectivamente; entretanto, apenas 53,1\% responderam à pergunta número 3, sendo os órgãos relatados como mais afetados pela doença olhos e rins. Além disso, 40,7\% dos entrevistados foram considerados aderentes ao tratamento. Em relação ao teste de Morisky, 51,9\% dos entrevistados foram considerados aderentes ao tratamento, apesar das respostas incorretas em relação ao esquecimento $(43,2 \%)$ e ao descuido do horário de utilização dos medicamentos $(29,6 \%)$.

Quadro 1 - Distribuição dos usuários que responderam corretamente aos questionamentos do teste de Batalla

\begin{tabular}{|l|c|c|}
\hline Questionamentos & N & (\%) \\
\hline (1) Qual a duração da doença & 61 & 75,3 \\
\hline (2) A doença pode ser controlada com dieta ou medicamentos & 74 & 91,4 \\
\hline (3) Cite dois órgãos que podem ser afetados pela doença & 43 & 53,1 \\
\hline Adesão & $\mathbf{3 3}$ & 40,7 \\
\hline
\end{tabular}

Fonte: Elaborado pelos autores (2018) 
Quadro 2 - Distribuição dos usuários que responderam corretamente aos questionamentos do teste de Morisky

\begin{tabular}{|l|c|c|}
\hline Questionamentos & N & (\%) \\
\hline (1) Você alguma vez se esqueceu de tomar o medicamento & 46 & 56,8 \\
\hline $\begin{array}{l}\text { (2) Você às vezes é descuidado com o horário para tomar seu me- } \\
\text { dicamento }\end{array}$ & 57 & 70,4 \\
\hline $\begin{array}{l}\text { (3) Quando você se sente melhor, às vezes, você para de tomar seu } \\
\text { medicamento }\end{array}$ & 75 & 92,6 \\
\hline $\begin{array}{l}\text { (4) Às vezes, se você se sente pior quando toma o medicamento, } \\
\text { você para de tomá-lo }\end{array}$ & 77 & 95,1 \\
\hline Adesão & $\mathbf{4 2}$ & $\mathbf{5 1 , 9}$ \\
\hline
\end{tabular}

Fonte: Elaborado pelos autores (2018).

$\mathrm{Na}$ análise das variáveis sociodemográficas e hábitos de vida, como possíveis fatores interferentes na adesão ao tratamento medicamentoso, pelo teste de Morisky houve significância estatística em relação à renda $(p<0,025)$. Verificou-se que os pacientes que recebem menos de 1 salário mínimo são menos aderentes (88,89\%) quando comparados aos pacientes que recebem de 1 a 2 salários mínimos. O tabagismo foi significante em relação à adesão avaliada pelo teste de Batalla $(p<0,033)$. Em relação às outras variáveis não houve nenhum tipo de associação significativa entre eles e a adesão em ambos os testes (Tabela 3).

Tabela 3 - Prevalência da adesão terapêutica pelo teste de Morisky segundo o perfil do usuário

Teste de Morisky

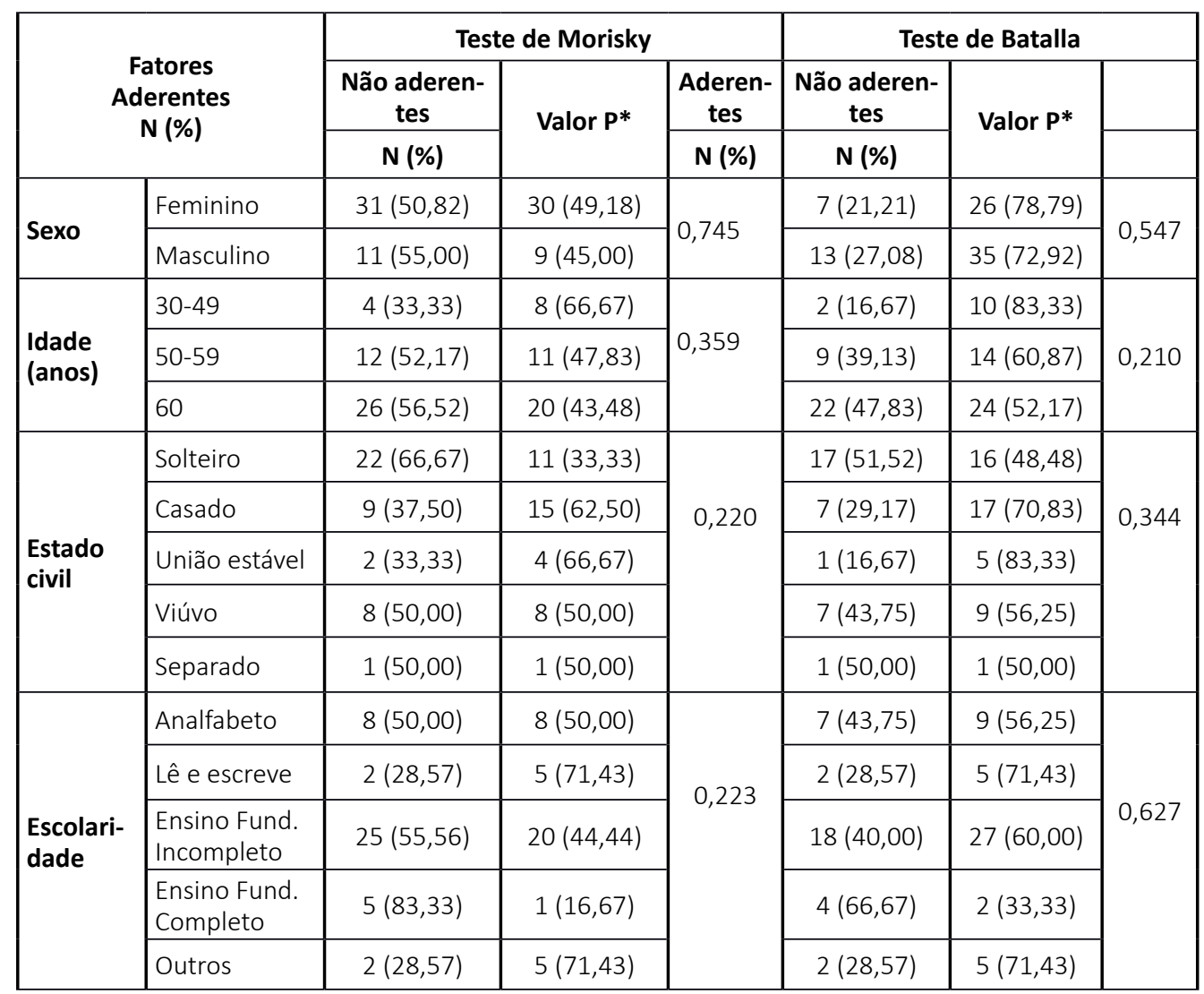




\begin{tabular}{|c|c|c|c|c|c|c|c|}
\hline \multirow{4}{*}{$\begin{array}{l}\text { Ocupa- } \\
\text { ção }\end{array}$} & Aposentado & $25(55,56)$ & $20(44,44)$ & \multirow{4}{*}{0,059} & $21(46,67)$ & $24(53,33)$ & \multirow{4}{*}{0,124} \\
\hline & Do lar & $10(50,00)$ & $10(50,00)$ & & $7(35,00)$ & $13(65,00)$ & \\
\hline & Doméstica & $4(100,00)$ & $0(0,00)$ & & $3(75,00)$ & $1(25,00)$ & \\
\hline & Outros & $3(25,00)$ & $9(75,00)$ & & $2(16,67)$ & $10(83,33)$ & \\
\hline \multirow{3}{*}{$\begin{array}{l}\text { Renda } \\
\text { (salário } \\
\text { mínimo) }\end{array}$} & $<1$ & $1(11,11)$ & $8(88,89)$ & \multirow{3}{*}{$0,025^{*}$} & $1(11,11)$ & $8(88,89)$ & \multirow{3}{*}{0,082} \\
\hline & $1-2$ & $39(58,21)$ & $28(41,79)$ & & $31(46,27)$ & $36(53,73)$ & \\
\hline & $<3$ & $2(40,00)$ & $3(60,00)$ & & $1(20,00)$ & $4(80,00)$ & \\
\hline \multirow{3}{*}{$\begin{array}{l}\text { Residen- } \\
\text { tes no } \\
\text { domicilio }\end{array}$} & 1 a 3 & $34(54,84)$ & $28(45,16)$ & \multirow{2}{*}{0,606} & $27(43,55)$ & $35(56,45)$ & \multirow{3}{*}{0,555} \\
\hline & 4 a 6 & $7(41,18)$ & $10(58,82)$ & & $5(29,41)$ & $12(70,59)$ & \\
\hline & 7 ou mais & $1(50,00)$ & $1(50,00)$ & & $1(50,00)$ & $1(50,00)$ & \\
\hline \multirow[t]{2}{*}{$\begin{array}{l}\text { Fuman- } \\
\text { tes }\end{array}$} & Sim & $3(100,00)$ & $0(0,00)$ & \multirow{2}{*}{0,089} & $3(100,00)$ & $0(0,00)$ & \multirow{2}{*}{$0,033^{*}$} \\
\hline & Não & $39(50,00)$ & $39(50,00)$ & & $30(38,46)$ & $48(61,54)$ & \\
\hline \multirow{2}{*}{$\begin{array}{l}\text { Consumo } \\
\text { de bebi- } \\
\text { da alcoó- } \\
\text { lica } \\
\end{array}$} & Sim & $2(100,00)$ & $0(0,00)$ & \multirow[t]{2}{*}{0,168} & $2(100,00)$ & $0(0,00)$ & \multirow{2}{*}{0,084} \\
\hline & Não & $40(50,63)$ & $39(49,37)$ & & $31(39,24)$ & $48(60,76)$ & \\
\hline \multirow{2}{*}{$\begin{array}{l}\text { Atividade } \\
\text { física }\end{array}$} & Sim & $16(50,00)$ & $16(50,00)$ & \multirow{2}{*}{0,787} & $14(43,75)$ & $18(56,25)$ & \multirow{2}{*}{0,656} \\
\hline & Não & $26(53,06)$ & $23(46,94)$ & & $19(38,78)$ & $30(61,22)$ & \\
\hline
\end{tabular}

${ }^{*} p<0,05$.

Fonte: Elaborada pelos autores (2018).

\section{DISCUSSÃO}

O perfil sociodemográfico dos participantes do estudo mostrou o predomínio do gênero feminino, na faixa etária de 60 anos ou mais. Em virtude da prevalência dessa faixa etária entre os entrevistados, a maior parte era formada por aposentados, que viviam com menos de 3 salários e possuíam Ensino Fundamental incompleto. O baixo nível de escolaridade pode, de certa forma, prejudicar a aprendizagem dos pacientes no que diz respeito a informações sobre o DM e à complexidade terapêutica, dificultando o sucesso do controle glicêmico, bem como a adesão ao tratamento. ${ }^{13}$

O envelhecimento da população é um fenômeno de amplitude mundial. No Brasil, a transição demográfica e epidemiológica modificou a pirâmide etária da população, elevou a expectativa de vida e aumentou a população idosa. A estimativa é que em 2050 existam cerca de 2 bilhões de pessoas com 60 anos ou mais. ${ }^{14} \mathrm{O}$ envelhecimento populacional desafia os sistemas de saúde pública e de previdência social, porém é importante buscar tecnologias que permitam o acesso da população aos melhores padrões de serviços de saúde, com vistas à redução da morbidade e mortalidade e melhora na qualidade de vida. ${ }^{15}$

Dos pacientes estudados, a maior parte não reside sozinho, o que poderia facilitar o seguimento da terapia medicamentosa e insulínica adequada, uma vez que os familiares poderiam estar auxiliando na administração dos medicamentos, minimizando erros e reduzindo os fatores de risco para a não adesão ao tratamento. 96,3\% relataram, entretanto, cuidar do próprio tratamento, o que pode dificultar o acompanhamento da terapêutica. Cabe ressaltar que os entrevista- 


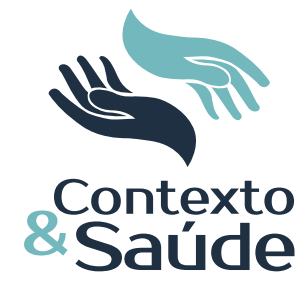

Editora Unijuí - Revista Contexto \& Saúde - ISSN 2176-7114 - v. 21, n. 44, out./dez. 2021

No que se refere às comorbidades associadas ao DM, a HAS apresenta alta prevalência em indivíduos com diabetes, chegando a ser duas vezes mais frequente em pacientes com diabetes em comparação com aqueles que não a possuem. ${ }^{21}$ Esse fato foi demonstrado também neste estudo, uma vez que, entre os 65 pacientes que apresentam alguma comorbidade, 93,8\% deles possuem hipertensão associada ao DM. Essa associação é preocupante, tendo em vista que a hipertensão nos diabéticos aumenta o risco de complicações cardiovasculares, como acidente vascular cerebral e aterosclerose. ${ }^{21}$

O uso de antidiabético oral foi evidenciado por todos os pacientes e a metformina e a glibenclamida são os medicamentos mais consumidos. ${ }^{22} \mathrm{~A}$ associação de um medicamento da classe das sulfonilureias com a metformina, é frequentemente utilizada no tratamento de pacientes com diabetes..$^{23}$ Os resultados obtidos favorecem as características de portadores de DM2, que representam cerca de $95 \%$ dos casos de diabetes, acometendo indivíduos em qualquer idade, porém com maior frequência em adultos. É uma doença de forte predisposição genética, quando muitos pacientes não apresentam sintomas clássicos da doença e podem permanecer durante anos sem diagnóstico, mas também fatores ambientais podem estar envolvidos. ${ }^{8}$

O teste padronizado de Batalla mensura a adesão por meio do nível de conhecimento do usuário sobre sua doença e o teste de Morisky mensura a adesão mediante as atitudes do paciente quanto à utilização dos medicamentos. A adesão terapêutica está sujeita à influência de múltiplos fatores que afetam diretamente o paciente. Quando não seguida corretamente pode afetar negativamente a evolução clínica do paciente e a sua qualidade de vida, constituindo-se em problema relevante, que pode trazer consequências pessoais, sociais e econômicas. $^{24}$

dos foram questionados somente em relação ao cuidador dos medicamentos e não quanto à ajuda familiar para uso dos medicamentos e aplicação da insulina.

Em relação à prevalência do uso do tabaco, os valores encontrados foram inferiores àqueles encontrados em outros estudos, ${ }^{16-17}$ nos quais prevaleceu um número maior de fumantes. O tabagismo é um fator de risco reconhecido para doenças cardiovasculares, uma das maiores complicações do diabetes, e seu combate deve fazer parte das estratégias de ação dos serviços de saúde. ${ }^{18}$ Quanto ao hábito do consumo de bebida alcoólica a prevalência foi baixa, em torno de $2,5 \%$. Pacientes com diabetes que apresentam sintomas causadas por neuropatias ou estados de hipoglicemias podem apresentar pioras dos sintomas ao fazer uso de bebida alcoólica. ${ }^{19}$

A maioria dos entrevistados $(60,5 \%)$ apresenta o sedentarismo como característica de vida, relatando não praticar nenhuma modalidade de atividade física. A atividade física é importante para a prevenção e tratamento do diabetes. A American Diabetes Association (ADA) recomenda 150 minutos de exercício aeróbico de intensidade moderada por semana e exercícios de resistência pelo menos duas vezes por semana em indivíduos com DM para melhorar a saúde geral. ${ }^{20}$ Cabe ressaltar que a mudança de hábitos e de estilo de vida dos indivíduos com diabetes deve ser enfatizada como parte da assistência terapêutica, pois ela contribui significativamente para a redução da glicemia. 


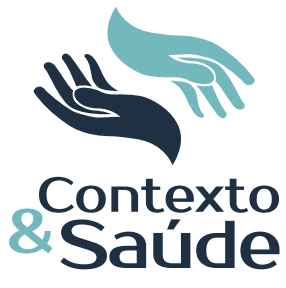

O conceito de adesão terapêutica varia entre diversos autores, mas, de forma geral, é compreendida como adesão quando o regime terapêutico prescrito é seguido em pelo menos $80 \%$ ou mais do seu total. ${ }^{25}$

A prevalência de adesão foi de $40,7 \%$ pelo teste de Batalla e $51,9 \%$ segundo o teste de Morisky, valores que se mostraram inferiores ao grau de adesão recomendado pela literatura, e corroboram outras investigações em que o mesmo instrumento foi utilizado. A falta de adesão ao tratamento é algo cada vez mais constatado, especialmente entre os portadores de doenças crônicas, nas quais se insere o DM. Essa baixa adesão à terapia farmacológica está associada ao maior risco de ocorrência de complicações cardiovasculares e hospitalizações. ${ }^{26}$

$\mathrm{O}$ esquecimento e o atraso no uso dos medicamentos são apontados como as principais causas para não adesão à terapêutica. Por se tratar de uma condição crônica, no diabetes os pacientes tendem a apresentar alterações psicossociais que podem prejudicar seu processo de aderência a um regime de tratamento vitalício que demanda tempo, recursos financeiros, em algumas situações, e o comprometimento de outras pessoas. ${ }^{27}$

Nesse sentido, há uma constante necessidade de intervenções educativas com vistas a orientar os pacientes portadores de diabetes quanto aos cuidados a serem tomados. Tais ações possibilitam uma visão crítica e uma maior participação e autonomia, potencializando a capacidade funcional dos pacientes para enfrentarem a complexidade em seus processos saúde/doença. ${ }^{28}$

A falta de informação da maioria dos entrevistados acerca da sua doença e tratamento, evidenciada pelo resultado do teste de Batalla, demostrou que o conhecimento pode influenciar o comportamento dos indivíduos ao tomar a decisão de seguir ou não a terapêutica prescrita para o diabetes.

$\mathrm{Na}$ avaliação dos pacientes pelo teste de Morisky as questões que apresentaram maior número de respostas "sim" foram quando questionados se esqueceram alguma vez de tomar seus medicamentos $(43,2 \%)$ e se descuidam quanto ao horário de tomá-los $(29,6 \%)$. Este descuido pode ter como consequência o surgimento das complicações decorrentes do diabetes, que podem ser ocasionadas tanto pelo excesso quanto pela falta do medicamento.

O desconhecimento dos indivíduos com diabetes sobre a importância do uso contínuo dos medicamentos para o controle do DM pode refletir em não adesão à terapia instituída, resultando em agravos que podem levar à morte. Salienta-se ainda ser essencial que os portadores conheçam as características da sua doença, considerando as particularidades de cada situação e isso cabe aos profissionais de saúde que acompanham esses pacientes informá-los. ${ }^{29}$

$\mathrm{Na}$ análise relacionando variáveis sociodemográficas e hábitos de vida como possíveis fatores interferentes na adesão ao tratamento medicamentoso, os resultados encontrados mostraram uma relação estatisticamente significativa entre o consumo do tabaco com a adesão ao tratamento pelo teste de Batalla. $\mathrm{O}$ tabagismo está associado a um risco elevado de doença cardiovascular e acidente vascular cerebral em pacientes com diabetes. Esse hábito tem participação em complicações microvasculares do diabetes, que incluem principalmente ne-

Editora Unijuí - Revista Contexto \& Saúde - ISSN 2176-7114 - v. 21, n. 44, out./dez. 2021 
fropatia, retinopatia e neuropatia, as quais podem ser desencadeadas por danos hiperglicêmicos a pequenos vasos sanguíneos..$^{18}$

No teste de Morisky ocorreu relação estatisticamente significativa da variável renda com a adesão. A renda é fator associado negativamente à adesão ao tratamento de doenças crônicas. Essa associação foi observada neste estudo, uma vez que os não aderentes tinham renda mensal menor que um salário mínimo. Essa relação pode ser justificada também pela falta de recursos financeiros para adquirir os medicamentos. ${ }^{30} \mathrm{O}$ acesso a medicamentos tem sido uma das pedras angulares para o controle do diabetes, principalmente pelo fato de os pacientes do estudo adquirirem os medicamentos pela rede pública. Os resultados são reflexos da necessidade em investimentos em políticas públicas que viabilizem melhores condições de educação, saúde e de vida aos pacientes diabéticos.

\section{CONCLUSÃO}

Os resultados revelaram a falta de informação da maioria dos pacientes atendidos no Centro de Saúde acerca de sua doença e tratamento, o que ocasiona uma falta de adesão à terapêutica, enfatizando a importância da comunicação entre os profissionais e os pacientes.

Além disso, o conhecimento do perfil sociodemográfico, hábitos de vida, tratamento e aspectos clínicos por parte dessa população é importante para elaborar estratégias direcionadas, visando à educação em saúde e minimização dos fatores que dificultam a adesão ao tratamento.

Desse modo, há uma necessidade em fortalecer as políticas de saúde com vistas a melhorar o acesso gratuito da população com doenças crônicas aos medicamentos para o tratamento do DM. Também é relevante a promoção de ações educativas interdisciplinares pelos serviços de saúde, como estratégias para melhorar o conhecimento e consequentemente a adesão dos pacientes ao tratamento.

Este estudo permitiu estimar a adesão/não adesão ao tratamento em uma amostra de pacientes com diabetes, contribuindo para gerar um corpo de evidências que dê suporte ao direcionamento de intervenções voltadas a esse grupo de pacientes.

\section{REFERÊNCIAS}

${ }^{1}$ Stokes A, Berry KM, Mchiza Z, Parker WA, Labadarios D, Chola L et al. Prevalence and unmet need for diabetes care across the care continuum in a national sample of South African adults: Evidence from the SANHANES-1, 2011-2012. PloS one. 2019;12(10):e0184264.

${ }^{2}$ Raposo JF. Diabetes na doença coronária: O risco do não diagnóstico. Acta medica portuguesa. 2017;30(6):429-430.

${ }^{3}$ Telo GH, Cureau FV, Souza MS, Andrade TS, Copês F, Schaan BD. Prevalence of diabetes in Brazil over time: a systematic review with meta-analysis. Diabetology \& metabolic syndrome. 2016;8(1):1-13.

${ }^{4}$ Saeedi P, Petersohn I, Salpea P, Malanda B, Karuranga S, Unwin N et al. Global and regional diabetes prevalence estimates for 2019 and projections for 2030 and 2045: Results from the International Diabetes Federation Diabetes Atlas. Diabetes research and clinical practice. 2019;157:107843. 


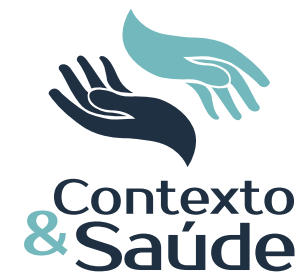

${ }^{5}$ Ministério da Saúde (BR). Secretaria e Vigilância em Saúde. Departamento de Análise de Situação de Saúde. Plano de ações estratégicas para o enfrentamento das doenças crônicas não transmissíveis (DCNT) no Brasil 2011-2022. Brasília (DF): Ministério da Saúde; 2011. [21 de setembro de 2020]. Disponível em: http://bvsms.saude.gov.br/ bvs/publicacoes/planoacoesenfrentdent2011.pdf

${ }^{6}$ Oliveira PSD, Bezerra EP, Andrade LLD, Gomes PLF, Soares MJGO, Costa MML. Practice nurse family health strategy in the prevention of diabetic foot. Revista de Pesquisa: Cuidado é Fundamental Online. 2016;8(3):4841-4849.

${ }^{7}$ Gregg EW, Sattar N, Ali MK. The changing face of diabetes complications. The Lancet Diabetes \& Endocrinology. 2016;4(6):537-547.

${ }^{8}$ Skyler JS, Bakris GL, Bonifacio E, Darsow T, Eckel RH, Groop L et al. Differentiation of diabetes by pathophysiology, natural history, and prognosis. Diabetes. 2017;66(2):241255.

${ }^{9}$ Raveendran AV, Chacko EC, Pappachan. Non-pharmacological treatment options in the management of diabetes mellitus. European Endocrinology. 2018;14(2):31.

${ }^{10}$ Silva WHD, Dantas DS, Nóbrega BSD, Queiroz MDSRD, Alves HDS. Evaluation of adherence to pharmacological treatment. Brazilian Journal of Pharmaceutical Sciences. 2019;55.

${ }^{11}$ Batalla C, Blanquer A, Ciurana R, García M, Jordi E, Pérez A. Cumplimiento de la prescripción farmacológica en pacientes hipertensos. Aten Primaria. 1984;1(4):185-91.

${ }^{12}$ Morisky DE, Green LW, Levine DM. Concurrent and predictive validity of a self-reported measure of medication adherence. Medical Care. 1986;67-74.

${ }^{13}$ Trevizan H, Bueno D, Koppitke L. Avaliação da adesão ao tratamento de pacientes usuários de insulina em uma unidade de atenção primária à saúde. Revista de APS. 2016;19(3).

${ }^{14}$ Morais AODDS, Oliveira AEFD, Matos CCDSA, Pessoa FS, Miranda MJS, Sgambatti MS. Saúde do idoso e a saúde da família. Cadernos de Saúde da Família; 2016.

${ }^{15}$ Miranda GMD, Mendes ADCG, Silva ALAD. O envelhecimento populacional brasileiro: desafios e consequências sociais atuais e futuras. Revista Brasileira de Geriatria e Gerontologia. 2016;19:507-519.

${ }^{16}$ Veloso J, Guarita-Souza LC, Lima Júnior EL, Ascari RA, Précoma DB. Perfil clínico de los pacientes con diabetes mellitus mediante intervenciones multidisciplinarias. Revista Cuidarte. 2020;11(3).

${ }^{17}$ Moreschi C, Rempel C, Siqueira DFD, Backes DS, Pissaia LF, Grave MTQ. Estratégias Saúde da Família: perfil/qualidade de vida de pessoas com diabetes. Revista Brasileira de Enfermagem. 2018;71:2899-2906.

${ }^{18}$ Campagna D, Alamo A, Di Pino A, Russo C, Calogero AE, Purrello F, Polosa R. Smoking and diabetes: dangerous liaisons and confusing relationships. Diabetology \& Metabolic Syndrome. 2019;11(1):1-12

${ }^{19}$ Munukutla S, Pan G, Deshpande M, Thandavarayan RA, Krishnamurthy P, Palaniyandi SS. Alcohol toxicity in diabetes and its complications: a double trouble? Alcoholism: Clinical and Experimental Research. 2016;40(4):686-697.

${ }^{20}$ Colberg SR, Sigal RJ, Yardley JE, Riddell MC, Dunstan DW, Dempsey PC et al. Physical activity/exercise and diabetes: a position statement of the American Diabetes Association. Diabetes Care. 2016;39(11):2065-2079.

${ }^{21}$ Petrie JR, Guzik TJ, Touyz RM. Diabetes, hypertension, and cardiovascular disease: clinical insights and vascular mechanisms. Canadian Journal of Cardiology. 2018;34(5):575584.

${ }^{22}$ Moreira PC, Silva LB, Petito G. Comorbidade entre Diabetes Mellitus e hipertensão arterial sistêmica em clientes de ESF de Ceres-Goiás. Revista Eletrônica da Faculdade de Ceres. 2015;4(2).

${ }^{23}$ Banik S, Hossain MS, Bhatta R, Akter M. Attenuation of lipid peroxidation and atherogenic factors in diabetic patients treated with gliclazide and metformin. Journal of research in medical sciences: the official journal of Isfahan University of Medical Sciences. 2018;23. 
${ }^{24}$ Dewulf NDLS, Monteiro RA, Passos ADC, Vieira EM, Troncon LEDA. Adesão ao tratamento medicamentoso em pacientes com doenças gastrintestinais crônicas acompanhados no ambulatório de um hospital universitário. Revista Brasileira de Ciências Farmacêuticas. 2006;42:575-584.

${ }^{25}$ Kleinsinger $F$. The unmet challenge of medication nonadherence. The Permanente Journal. 2018;22.

${ }^{26}$ Han E, Suh DC, Lee SM, Jang S. The impact of medication adherence on health outcomes for chronic metabolic diseases: a retrospective cohort study. Research in Social and Administrative Pharmacy. 2014;10(6):e87-e98.

${ }^{27}$ Silvano GP, Jaques Júnior NP, Machado MO. Perfil farmacoepidemiológico dos pacientes diabéticos participantes do programa Hiperdia em Tubarão-SC, Brasil Pharmacoepidemiological profile of diabetic patients participants of Hiperdia program in Tubarão-SC, Brazil. Journal of Health \& Biological Sciences. 2016;4(2):102-110.

${ }^{28}$ Marques MB, Coutinho JFV, Martins MC, Lopes MVDO, Maia JC, Silva MJD. Intervención educativa para la promoción del autocuidado de ancianos con diabetes mellitus. Revista da Escola de Enfermagem da USP. 2019;53.

${ }^{29}$ Santos FS, Oliveira KR, Colet CF. Adesão ao tratamento medicamentoso pelos portadores de Diabetes Mellitus atendidos em uma Unidade Básica de Saúde no município de Ijuí/RS: um estudo exploratório. Journal of Basic and Applied Pharmaceutical Sciences. 2010;31(3)

${ }^{30}$ Gewehr DM, Bandeira VAC, Gelatti GT, Colet CDF, Oliveira KRD. Adesão ao tratamento farmacológico da hipertensão arterial na Atenção Primária à Saúde. Saúde em Debate. 2018;42:179-190. 